The researchers found that 147 patients (29.9\%) received inadequate antimicrobial treatment for their bloodstream infections. The hospital mortality rate of patients with a bloodstream infection receiving inadequate antimicrobial treatment $(61.9 \%)$ was statistically greater than the hospital mortality rate of patients with a bloodstream infection who received adequate antimicrobial treatment (28.4\%). Multiple logistic regression analysis identified the administration of inadequate antimicrobial treatment as an independent determinant of hospital mortality. The most commonly identified bloodstream pathogens and their associated rates of inadequate antimicrobial treatment included vancomycinresistant enterococci $(\mathrm{n}=17 ; 100 \%)$, Candida species $(\mathrm{n}=41$; 95.1\%), oxacillin-resistant Staphylococcus aureus $(\mathrm{n}=46$; $32.6 \%$ ), coagulase-negative staphylococci $(n=96 ; 21.9 \%)$, and Pseudomonas aeruginosa $(\mathrm{n}=22 ; 10.0 \%)$. A statistically significant relation was found between the rates of inadequate antimicrobial treatment for individual microorganisms and their associated rates of hospital mortality. Multiple logistic regression analysis also demonstrated that a bloodstream infection attributed to Candida species, prior administration of antibiotics during the same hospitalization, decreasing serum albumin concentrations (1-g/dL decrements), and increasing central catheter duration (1-day increments) were independently associated with the administration of inadequate antimicrobial treatment.

The authors suggest that clinical efforts should be aimed at reducing the administration of inadequate antimicrobial treatment to hospitalized patients with bloodstream infections, especially individuals infected with antibiotic-resistant bacteria and Candida species.

FROM: Ibrahim EH, Sherman G, Ward S, Fraser VJ, Kollef MH. The influence of inadequate antimicrobial treatment of bloodstream infections on patient outcomes in the ICU setting. Chest 2000;118:146-155.

\section{Disinfectant Contaminated With Klebsiella axytoca-Source of Sepsis in Babies}

An outbreak of sepsis in an ICU in Giessen, Germany, has been traced to the use of formaldehyde-based disinfectant contaminated with Klebsiella oxytoca. Two of the children died, and one of the survivors was severely disabled. Reiss and colleagues from Justus-Liebig University, Glessen, Germany, analyzed blood cultures from the 28 infants affected between October 1996 and March 1999. Enzyme and antibiotic patterns were identical to those of bacterial isolates obtained from the plastic buckets where the disinfectant was stored.

Three months before the outbreak, the disinfectant concentration had been lowered from the recommended concentration of $0.5 \%$ to $0.25 \%$ because of complaints of skin irritation by hospital staff. The higher concentration of disinfectant consistently killed $K$ oxytoca within a few minutes, whereas the $0.25 \%$ solution of disinfectant supported the growth of $K$ oxytoca at room temperature, with the microbial population doubling every 20 hours The authors report that no further cases of sepsis due to $K$ oxytoca have occurred since the following infection control measures were adopted in the ICU: (1) increasing the concentration of disinfectant to $0.5 \%$ and (2) replacing plastic pails that held the diluted disinfectant with metal pails, which were autoclavable.

FROM: Reiss I, Borkhardt A, Fussle R, Sziegoleit A, Gortner L. Disinfectant contaminated with Klebsiella oxytoca as a source of sepsis in babies. Lancet 2000;356:310-311.

\section{Epidemiology of Bacteriuria Caused by VRE}

Wong and coinvestigators recently reported on a retrospective study on the epidemiology of vancomycin-resistant enterococcal (VRE) bacteriuria. Their objectives were to describe the frequency of VRE bacteriuria, to use strict definitions to distinguish symptomatic urinary tract infection (UTI) versus urine colonization without pyuria versus asymptomatic bacteriuria with pyuria, and to describe the outcomes of each group.

During the 18-month study period, 98 (92\%) of the 107 patients with urine cultures positive for VRE $(23 / 10,000$ admissions) had charts available for review. Enterococcus faecium was recovered in 94 of 98 patients; in the remaining 4 patients, Enterococcus faecalis was recovered. Thirtyseven patients were colonized with VRE; 21 patients had asymptomatic bacteriuria, and the status of 27 patients was not ascertainable. Thirteen patients had VRE UTIs, with two associated bacteremias and one death. Patients with UTI versus patients without UTI were more likely to have an underlying malignancy $(39 \%$ vs $9 \%, P=.014)$.

The authors concluded that the majority of urine cultures yielding VRE do not represent true infection, rather colonization or asymptomatic bacteriuria.

FROM: Wong AH, Wenzel RP, Edmond MB. Epidemiology of bacteriuria caused by vancomycin-resistant enterococci-a retrospective study. Am J Infect Control 2000;28:277-281.

\section{VRE Risk Factors Among Renal Patients}

Beltrami and coinvestigators, from the CDC's Hospital Infections Program and Community Hospital East in Indianapolis, Indiana, conducted a study on risk factors associated with vancomycin-resistant enterococci (VRE) among patients on a renal ward during a community hospital outbreak. During an outbreak of VRE infection and colonization at a community hospital in Indianapolis, they performed a case-control study of patients on the hospital's renal unit to determine risk factors for acquisition of VRE among this potentially high-risk patient population.

Twenty-four renal patients with VRE colonization or infection (ie, case-patients) were compared by univariate and multivariate analyses with 29 renal patients with nosocomially acquired vancomycin-susceptible enterococcal infection and colonization (ie, controls). The results showed that age and length of hospitalization were similar between 